\title{
Spherical symmetry breaking in electric, magnetic and toroidal multipole moment radiations in spherical toroidal resonant cavities and optimum-efficiency antennas
}

\author{
E. Ley-Koo ${ }^{a}, \mathrm{H}$. Torres-Bustamante ${ }^{a}$, and A. Góngora T. ${ }^{b}$ \\ ${ }^{a}$ Instituto de Física, Universidad Nacional Autónoma de México, Ciudad de México, México. \\ ${ }^{b}$ Formely of Northeastern University, Boston Massachusetts, USA.
}

Received 31 October 2020; accepted 21 February 2021

This Letter reports the breaking of the spherical symmetry in the complete electromagnetic multipole expansion when its sources are distributed on spherical toroidal surfaces, identifying the specific geometrical and physical changes from the familiar case of sources on a spherical surface. In fact, for spherical toroids defined by concentric spherical rings and symmetric conical rings, the boundary conditions at the latter are not compatible in general with integer values for the orbital angular momentum label of the multipole moments: the polar angle eigenfunctions become Legendre functions of order $\lambda$ and associativity $\mathrm{m}$ represented as infinite series with a definite parity, and their complementary associated radial functions are spherical Bessel functions of the same order $\lambda$. Consequently, the corresponding multipole sources for the electric, magnetic and toroidal moments and their connections are identified within the Debye formalism, and the appropriate outgoing wave Green functions are constructed in the new basis of eigenfunctions of the Helmholtz equation. Our familiarity with the exact solutions, for the cases of the complete sphere and of cylindrical toroids, allow us to give a preliminary account of the electromagnetic fields for the spherical toroids via the integration of their sources and the Green function for resonant cavities and optimum-efficiency antennas.

Keywords: Electromagnetic multipole moment expansion; symmetry breaking from sphere to spherical toroids; longitudinal; poloidal and toroidal sources and fields for electric; magnetic and toroidal moments; and their connections in Debye formalism; Green functions for resonant cavities and optimum-efficiency antennas.

PACS: $41.20 \mathrm{Jb}$

DOI: https://doi.org/10.31349/RevMexFis.67.174

\section{Introduction}

This Letter is motivated by the observations of toroidal dipole interactions in metamaterials [1,2] and nanomaterials [3], and attempts to establish the difference between electric and toroidal moment electromagnetic radiations using the familiar spherical multipole expansions in the far-away zone [4]. The works in [1-3] and [5,6] have cited our work [7] on the complete electromagnetic multipole expansion including toroidal moments, which is complete on two accounts: 1) it describes the fields inside and outside a spherical toroidal surface where the sources are confined, and 2) it includes the toroidal moments. The use of the multipole expansion in the far-away zone ignores the roles of the sources and of Maxwell's equations in the boundary condition form, which are indispensable to connect the inner and outer solutions satisfying the homogeneous Helmholtz equation. The citations of our work motivated us to revisit it [8], and we have just realized that when going from sources confined on a spherical surface [9] to sources confined on a spherical toroidal surface, the spherical symmetry of the first one is broken in the second one. Consequently, this contribution is focused on the manifestations of the symmetry breaking and its general consequences, which hopefully will be particularly useful for the metamaterials and nanomaterials communities. We may add that we have just completed the identification of the complete and exact solutions of a family of electromagnetic multipole moments in cylindrical toroids as reported in [10], which has been an important point of reference and guidance in our writing of this Letter. It is also important to mention that Refs. [1-3] were reviewed in [11], recognizing that the ideas of Superintegrability and Symmetry Breaking apply not only for atoms and molecules, but also to the electromagnetic multipole expansion, when its sources are confined on toroidal surfaces.

The confinement of the sources and Dirichlet and Neumann boundary conditions for resonant cavities and optimum-efficiency antennas, respectively, lead to quantization of wave numbers and frequencies, and to complete and orthonormal sets of eigenfunctions. Symmetry breaking involves the same constants of motion but different eigenvalues: $\ell$ becomes $\lambda$ with non integer values. Correspondingly, the associated Legendre Polynomials become Legendre functions with $(\lambda, m)$ parameters in their hypergeometric function representation, with a well defined parity for symmetric toroids. The radial spherical functions become $R_{\lambda}$, wellbehaved superpositions of spherical Bessel functions $j_{\lambda}$ and $n_{\lambda}$ inside the toroid, and outside $j_{\lambda}$ in the hole and $h_{\lambda}^{(1)}$ outgoing spherical wave.

The longitudinal, toroidal and poloidal current densities of a given multipolarity, on each section of the spherical toroidal surface, are constructed from a common scalar Debye potential involving one eigenfunction of the sets mentioned above, via the application of the gradient $\nabla$, angular 
momentum $\mathbf{L}=-i \mathbf{r} \times \boldsymbol{\nabla}$ and curl $\nabla \times$ operators, in the succession already identified for cylindrical toroids [10]. They are the sources of the electric intensity fields in the inhomogeneous Helmholtz equation. The complementary sources of the magnetic induction field in the same equation are also identified.

The Green functions for the spherical toroids are also constructed using the bases of the above-mentioned radial and polar angle eigenstates, as the counterpart of those in [10]. In [7] the point unit charge Green function was used, with the sources of Eqs. (23) and (24) involving Heaviside step functions, representing uniform distributions on each section of the spherical toroidal surface. It is appropriate at this point to recognize that the orthonormality and completeness of the radial and polar angle eigenfunctions at the boundary defined by the other variable provide the representations of the difference of the respective Dirac-delta functions, and that their integrations become the difference of the Heaviside step functions. These relationships are important to understand the connections between the Green functions and sources in $[7,8]$ and this contribution, as well as the equivalence of their integrated results.

Consequently, the successive sections of this Letter in 2. Spherical Symmetry Breaking, explain the reasons behind it and identify its explicit manifestations, 3. Sources Distributed on the Surfaces of Spherical Toroids, identify them from a common Debye scalar potential for the successive electric, magnetic and toroidal moments on the basis of their multipolarities and directionalities, including their successive connections, 4. Scalar Green Functions, to be constructed using the solutions of the homogeneous Helmholtz equations inside and outside the toroidal surface, satisfying Dirichlet boundary conditions for resonant cavities and Neumann boundary conditions for optimum-efficiency antennas, respectively, and 5. Discussion of the anticipated results for the electromagnetic fields, to be evaluated from the integrations of the sources in Sec. 3 and the Green functions in Sec. 4. The Appendix serves as a guide to the readers interested in understanding the details of the text.

\section{Spherical symmetry breaking}

The restriction of distributing the sources on the surface of a spherical toroid formed by spherical concentric rings of radius $r=a$ and $r=b$, and symmetric conical rings at the polar angles $\theta=\theta_{1}$ y $\theta_{2}=\pi-\theta_{1}$, and $0<\varphi<2 \pi$, in spherical coordinates, allows the construction of solutions of Maxwell equations using the basis of solutions for the scalar homogeneous Helmholtz equation, inside and outside the toroid, as Debye potentials subject to Dirichlet and Neumann boundary conditions for the electromagnetic radiation fields in resonant cavities and optimum-efficiency antennas, respectively. In general, for arbitrary values of the polar angle $\theta_{1}$, the above mentioned boundary conditions can not be satisfied by the familiar associated Legendre polynomials of degree $(\ell, m)$. The quantum label $\ell$ takes non integer values $\lambda$, and the polynomials are replaced by Legendre functions of order $\lambda$, associativity $m$, and definite parity, represented as infinite series superpositions of hypergeometric functions with arguments $(1-\cos \theta) / 2$ and $(1+\cos \theta) / 2$, with common labels in $\lambda$ and $m=1$ :

$$
\begin{aligned}
\Theta_{\lambda}^{1}(\cos \theta) & =N_{\lambda}^{1} \sin \theta \frac{1}{\sqrt{2}} \\
& \times\left({ }_{2} F_{1}\left[-\{\lambda-1\}, \lambda+2,1 ; \frac{1-\cos \theta}{2}\right] \pm{ }_{2} F_{1}\right. \\
& {\left.\left[-(\lambda-1), \lambda+2,1 ; \frac{1+\cos \theta}{2}\right]\right) }
\end{aligned}
$$

The order $\lambda$ is determined by the nodes and the extreme points of the series in $\theta_{1}$. The companion radial functions are superpositions of the corresponding ordinary and Neumann type spherical Bessel functions inside the toroid $r \in[a, b]$ :

$$
R_{\lambda}(k r)=A_{\lambda}(k) j_{\lambda}(k r)+B_{\lambda}(k) n_{\lambda}(k r)
$$

ordinary spherical Bessel function in the hole of the toroid $r \in[0, a]$, and outgoing wave spherical Bessel function outside $r \in[b, \infty]$. The respective boundary conditions on the radial eigenfunctions at the inner and outer spherical rings can be satisfied only for specific choices of coefficients $A_{\lambda}(k), B_{\lambda}(k)$, leading to resonant wave numbers and their associated frequencies, which turn out to be interlaced for the successive modes of the cavities and antennas. Additionally, their nodal lines combined with those of their companion polar eigenfunctions determine the positions of foursided perimeters in each meridian plane; as well as the corresponding positions of extreme amplitudes inside the perimeter. The product of the radial and polar eigenfunctions apply to the magnetic moments. For the electric and toroidal moments there is an extra radial factor, argument of the spherical Bessel functions, as justified at the end of Sec. 3. Reference [11] illustrates the symmetry breaking in the context of confined atoms and molecules, and also included reviews of [13].

\section{Sources distributed on the surfaces of spherical toroids}

The sources of the electromagnetic fields distributed on the surface of the spherical toroids are identified from a common Debye scalar potential, for each spherical and conical ring sections at the respective positions $r=a, b$ and $\theta=\theta_{1}, \theta_{2}$, respectively; with multipole distributions and directionalities to be described next for the successive multipole moments. For the electric moments, the starting point is the longitudinal current density with a gradient of the Debye scalar potential 
of the chosen multipolarity:

$$
\begin{aligned}
\mathbf{K}^{E l} & =\kappa_{r_{0}} \hat{r}\left(\frac{d}{d r} R_{\lambda}(k r)\right)\left[Y_{\lambda 0}\left(\theta_{1}, \phi\right)+Y_{\lambda 0}\left(\theta_{2}, \phi\right)\right] \\
& +\kappa_{\theta_{0}} \frac{\hat{\theta}}{r}\left(\frac{d}{d \theta} Y_{\lambda 0}(\theta, \phi)\right)\left[R_{\lambda}(k a)+R_{\lambda}(k b)\right] .
\end{aligned}
$$

Its divergence leads to the surface charge density via the continuity equation,

$$
\begin{aligned}
\boldsymbol{\nabla} \cdot \mathbf{K}^{E l} & =i \omega \sigma=\kappa_{r_{0}}\left(\frac{1}{r^{2}} \frac{d}{d r}\left[r^{2} R_{\lambda}(k r)\right]\right) \\
& \times\left[Y_{\lambda 0}\left(\theta_{1}, \phi\right)+Y_{\lambda 0}\left(\theta_{2}, \phi\right)\right] \\
& +\kappa_{\theta_{0}} \frac{1}{r^{2}}\left(\frac{1}{\sin \theta} \frac{d}{d \theta}\left[\sin \theta \frac{d}{d \theta} Y_{\lambda 0}(\theta, \phi)\right]\right) \\
& \times\left[R_{\lambda}(k a)+R_{\lambda}(k b)\right] .
\end{aligned}
$$

In turn, the cross product of $(r \sin \theta) \hat{\varphi}$ and $(\mathbf{r}+r \hat{\theta})$ with the longitudinal current density leads to the poloidal current density $\mathbf{K}^{E p}$ of the electric intensity field, and the source density $\mathbf{S}^{E t}$ of the magnetic induction, in the respective Helmholtz equations:

$$
\begin{aligned}
\mathbf{K}^{E p} & =\kappa_{r_{0}} \hat{\theta}\left(r \frac{d}{d r} R_{\lambda}(k r)\right) \sin \theta\left[Y_{\lambda 0}\left(\theta_{1}, \phi\right)+Y_{\lambda 0}\left(\theta_{2}, \phi\right)\right] \\
& -\kappa_{\theta_{0}} \hat{r}\left(\sin \theta \frac{d}{d \theta} Y_{\lambda 0}(\theta, \phi)\right)\left[R_{\lambda}(k a)+R_{\lambda}(k b)\right] \\
\mathbf{S}^{E t} & =\hat{\varphi}\left(-\kappa_{r_{0}}\left[r \frac{d}{d r} R_{\lambda}(k r)\right]\left[Y_{\lambda 0}\left(\theta_{1}, \phi\right)+Y_{\lambda 0}\left(\theta_{2}, \phi\right)\right]\right. \\
& \left.+\kappa_{\theta_{0}}\left[\frac{d}{d \theta} Y_{\lambda 0}(\theta, \phi)\right]\left[R_{\lambda}(k a)+R_{\lambda}(k b)\right]\right) .
\end{aligned}
$$

In turn, the curl of $\mathbf{K}^{E p}$ becomes the toroidal current density for the magnetic moments $\mathbf{K}^{M t}$ as the source density of the electric intensity field:

$$
\begin{aligned}
\mathbf{K}^{M t} & =\frac{\hat{\varphi}}{r}\left(\kappa_{r_{0}}\left[\frac{d}{d r}\left\{r^{2} \frac{d}{d r} R_{\lambda}(k r)\right\}\right] \sin \theta\right. \\
& \times\left[Y_{\lambda 0}\left(\theta_{1}, \phi\right)+Y_{\lambda 0}\left(\theta_{2}, \phi\right)\right] \\
& +\kappa_{\theta_{0}}\left[R_{\lambda}(k a)+R_{\lambda}(k b)\right] \\
& \left.\times\left[\frac{d}{d \theta}\left\{\sin \theta \frac{d}{d \theta} Y_{\lambda 0}(\theta, \phi)\right\}\right]\right) .
\end{aligned}
$$

Additionally, the curl of $\mathbf{K}^{M t}$ leads to the poloidal source density $\mathbf{S}^{M p}$ of the magnetic induction field:

$$
\begin{aligned}
\mathbf{S}^{M p} & =\left(-\kappa_{r_{0}} \hat{\theta}\left[\frac{1}{r} \frac{d}{d r}\left\{\frac{d}{d r}\left(r^{2} \frac{d}{d r} R_{\lambda}(k r)\right)\right\}\right] \sin \theta\right. \\
& \times\left[Y_{\lambda 0}\left(\theta_{1}, \phi\right)+Y_{\lambda 0}\left(\theta_{2}, \phi\right)\right] \\
& +\kappa_{\theta_{0}} \hat{r} \frac{1}{r^{2}}\left[R_{\lambda}(k a)+R_{\lambda}(k b)\right] \\
& \left.\times\left[\frac{1}{\sin \theta} \frac{d}{d \theta}\left\{\sin \theta \frac{d}{d \theta}\left(\sin \theta \frac{d}{d \theta} Y_{\lambda_{0}}(\theta, \phi)\right)\right\}\right]\right) .
\end{aligned}
$$

Similarly, the curl of $\mathbf{S}^{E t}$ is identified as the poloidal current density of the toroidal moments $\mathbf{K}^{T p}$, source of $\mathbf{E}^{T p}$ :

$$
\begin{aligned}
\mathbf{K}^{T p} & =\left(\kappa_{r_{0}} \hat{\theta}\left[\frac{1}{r} \frac{d}{d r}\left\{r^{2} \frac{d}{d r} R_{\lambda}(k r)\right\}\right]\right. \\
& \times\left[Y_{\lambda 0}\left(\theta_{1}, \phi\right)+Y_{\lambda 0}\left(\theta_{2}, \phi\right)\right] \\
& +\kappa_{\theta_{0}} \frac{\hat{r}}{r}\left[R_{\lambda}(k a)+R_{\lambda}(k b)\right] \\
& \left.\times\left[\frac{1}{\sin \theta} \frac{d}{d \theta}\left\{\sin \theta \frac{d}{d \theta} Y_{\lambda 0}(\theta, \phi)\right\}\right]\right) .
\end{aligned}
$$

Additionally, the curl of $\mathbf{K}^{T p}$ yields the toroidal source density $\mathbf{S}^{T t}$ for $\mathbf{B}^{T t}$ :

$$
\begin{aligned}
\mathbf{S}^{T t} & =\frac{\hat{\varphi}}{r}\left(\kappa_{r_{0}}\left[\frac{d}{d r}\left\{\frac{d}{d r}\left(r^{2} \frac{d}{d r} R_{\lambda}(k r)\right)\right\}\right]\right. \\
& \times\left[Y_{\lambda 0}\left(\theta_{1}, \phi\right)+Y_{\lambda 0}\left(\theta_{2}, \phi\right)\right] \\
& -\kappa_{\theta_{0}} \frac{1}{r}\left[R_{\lambda}(k a)+R_{\lambda}(k b)\right] \\
& \left.\times\left[\frac{d}{d \theta}\left\{\frac{1}{\sin \theta} \frac{d}{d \theta}\left(\sin \theta \frac{d}{d \theta} Y_{\lambda 0}(\theta, \phi)\right)\right\}\right]\right)
\end{aligned}
$$

Notice that the longitudinal, poloidal and toroidal source densities involve first derivatives of the radial and polar angle eigenfunctions, for the electric moments. The curls of the last two transverse current densities yield the toroidal and poloidal current densities, for the magnetic and toroidal moments, respectively, involving second derivatives associated with the Laplace operator, and yielding the radial and polar eigenvalues and eigenfunctions of the Helmholtz equation. In turn, the additional curls of the current densities lead to the source densities for the respective magnetic induction fields, with third order derivatives in the radial and polar angle eigenfunctions. These dependences are behind the difference by a factor of $k^{2}$ between the electric and toroidal moment electromagnetic fields, sharing otherwise the same space dependences in their transverse fields. as well as their resonant frequencies for cavities and antennas. On the other hand, the electromagnetic fields for magnetic moments differ from the other two by exchange of the directionalities of their electric intensity and magnetic induction fields, and even versus odd orders of derivatives, as well as different resonance frequencies. In the case of electric moments, it is important to point out the presence of the common radial factor in the vectors $\mathbf{r}, r \hat{\theta}$ and $r \sin \theta \hat{\varphi}$, which via their successive vector products with the longitudinal current density yield the respective components of the toroidal source of $\mathbf{B}^{E t}$ and the poloidal current source of $\mathbf{E}^{E p}$. The extra factor of the spherical Bessel functions mentioned in Sec. 2 for electric and toroidal moments is thus justified. 


\section{Green functions}

This section contains the scalar Green functions of the Helmholtz equation with a unit charge at the point $\mathbf{r}^{\prime}$, on the toroidal surface, using the inner solutions of the homogeneous equation, Eqs. (1) and (2), satisfying the Dirichlet boundary condition for the resonant cavities, and the Neumann boundary conditions for the optimum-efficiency antennas, as well as for the near fields produced by the longitudinal current and charge densities in the electric moments:

$$
\begin{aligned}
\mathbf{G}^{(+)}\left(\mathbf{r}, \mathbf{r}^{\prime}\right) & =\sum_{m} \sum_{n_{\theta}} \sum_{n_{r}} g_{n_{r} n_{\theta}}(k) R_{\lambda_{n_{\theta}}}\left(k r_{\mathrm{int}}\right) h_{\lambda_{n_{\theta}}}^{(1)}\left(k r_{\mathrm{ext}}\right) \\
& \times \Theta_{\lambda_{n_{\theta}}}^{1}(\cos \theta) \Theta_{\lambda_{n_{\theta}}}^{1}\left(\cos \theta^{\prime}\right) \frac{e^{i m\left(\varphi-\varphi^{\prime}\right)}}{2 \pi} . \quad(11)
\end{aligned}
$$

The outgoing Green function in (11) uses the same angular bases and the linear superposition of the products of the inner and outer Bessel functions with coefficients $g_{n_{r} n_{\theta}}(k)$, taking into account the quantization and order of excitation in the respective degrees of freedom. The coefficients are evaluated by integrating the radial part of the Helmholtz equation involving the Wronskian of the inner and outer spherical Bessel functions:

$$
\begin{aligned}
g_{n_{r} n_{\theta}}(k) & =\frac{-4 \pi}{k r^{2} \mathcal{W}\left(R_{\lambda}(k r), h_{\lambda}^{(1)}(k r)\right)} \\
& =\frac{-4 \pi k}{\left(i A_{\lambda}(k)-B_{\lambda}(k)\right)} .
\end{aligned}
$$

\section{Discussion}

This section describes the anticipated results for the electromagnetic fields to be obtained from the integration of the source densities identified in Sec. 3 and the Green functions of Sec. 4, for the successive magnetic, toroidal and electric moments. This order is followed because the first two share transverse sources and only radiation fields, while the last one also involves the longitudinal current and charge densities and their near fields in the vicinity of the spherical toroid. The presence of the latter illustrates clearly the difference between the toroidal and electric moments.

For the magnetic moments, the toroidal surface current densities $\mathbf{K}^{M t}$ yield the toroidal electric intensity field $\mathbf{E}(r, \theta, \varphi)$, while their poloidal curls, $\mathbf{S}^{M p}$, yield the poloidal magnetic induction fields $\mathbf{B}(r, \theta, \varphi)$, for the interior of the resonant cavities and for the interior and exterior of the toroidal optimum-efficiency antennas, respectively. The vanishing of the radial functions inside the toroidal surface at the inner and outer spherical rings of radii $a$ and $b$, required by the Dirichlet boundary condition, guarantees that the outer fields vanish in the first case. On the other hand, in the second case, the extensions of the radial functions with Neumann boundary conditions to the outer intervals, $[0, a]$ in the hole and $[b, \infty]$ to the far-away zone, guarantee their proper behaviors in their respective domains. In both cases, the electric intensity field turns out to be consistent with Gauss' and Faraday's laws, while the magnetic induction field is also consistent with Gauss', Ampere's and Maxwell's laws in their boundary condition forms. Additionally, their field lines may be written in their differential forms and turn out to be integrable for the poloidal fields, yielding their vortex structure in each meridian plane within four-sided radial and circular separatrices.

For the toroidal moments, the poloidal current densities $\mathbf{K}^{T p}$ yield the poloidal electric intensity fields $\mathbf{E}^{M p}$, while the toroidal source $\mathbf{S}^{T t}$ yields the toroidal magnetic induction field $\mathbf{B}^{M t}$. Notice the changes in directionalities of the respective sources and fields when going from the magnetic multipoles to the toroidal ones, directly connected with the connections of the fields themselves via Faraday and Maxwell laws in both cases. This observation is pertinent here, in order to avoid the repetition of the discussion of the last part in the previous paragraph.

For the electric moments, the difference with respect to the toroidal moments, besides the extra $k^{2}$ in $\mathbf{B}^{M t}$ compared to $\mathbf{B}^{E t}$, is obviously the presence of the longitudinal current density and its associated charge density. The evaluation of the longitudinal electric field from the integration of the longitudinal current density and the respective Green functions for the resonant cavities and antennas, $\mathbf{E}^{E l}$, yields the surface charge density distribution at the toroidal surface via Gauss's law in its boundary condition form; on the other hand, its normal components at the first separatrices of the radiation fields, inside and outside the toroid, vanish at the separatrices becoming tangential near them, and consistent with Faraday's law. The reader may see their counterparts for the sphere in [9], and for circular cylindrical toroids in [10].

The analytical elements described in the successive sections are the basis for the numerical computations of the respective electromagnetic fields, for specific values of the geometrical parameters of the spherical toroids. The corresponding results will be reported in the near future.

The comparison of the contents of this Letter and our previous works may help the readers to appreciate the differences in them. In fact, the Letter is the natural extension of [7,8], recognizing explicitly the spherical symmetry breaking and its implementation. The comparison with [9] shows their common structure allowing for the difference in their respective inner and outer solutions. In the comparison with [10], its separation into independent axial and circular radial components is replaced by the separation into spherical radial and common polar angle components; in the first one, both degrees of freedom determine the resonant frequencies, and in the second one, the spherical radial component determines by itself the resonant frequencies.

We conclude this Letter by pointing out that its contents provide the new elements of spherical symmetry breaking eigenfunctions of the scalar homogeneous Helmholtz equations, the connections between the sources of the successive electromagnetic multipole fields for electric, magnetic and toroidal moments, and the respective Green functions for 
spherical toroidal resonant cavities and optimum-efficiency antennas, needed for the evaluation of the respective electric intensity and magnetic induction fields. One of its important results is the identification of the difference between the toroidal and electric moments in the presence of the localized near field in the latter produced by the longitudinal current and its associated charge density. Hopefully, this will be useful and of interest for the Metamaterials and Nanomaterials communities, by providing exact results to compare with their different interpretations of the toroidal moments, as illustrated in [4].

\section{Appendix A.}

The additional material in this Appendix, included for the benefit of a wider readership of Revista Mexicana de Fisica interested in this Letter, is presented in two complementary parts: 1) A guide to the References, in connection with the motivation for writing the Letter, its antecedents and new results; and 2) The Debye formalism and its application needed to understand the results reported in Sec. 2-5.

1) As explained in the Introduction, the motivation of this Letter is associated with the citations of our work [1] in [1$3]$, reporting the observations of toroidal dipole interactions in metamaterials and nanophotonics, respectively. Our work [11] included reviews of reports related to [1-3]. Additional reports on toroidal moments [4-6] show different interpretations about them. Our additional works on the electromagnetic moments with sources confined on spherical, spherical toroidal and cylindrical toroidal surfaces [8-10] are the immediate antecedents of the Letter. The ideas of superintegrability and symmetry breaking in [11] are applicable for atoms and molecules, and also for electromagnetic fields. Superintegrability of a wave equation implies separability and integrability in more coordinate systems than degrees of freedom. Symmetry breaking in [11] and the Letter is due to the change from the spherical boundary [9] to the spherical toroidal boundary [7,9], with the consequences described in Secs. 2-5. Each Reader may find in the respective sets of Refs. [1-6] and [7-11], the detailed antecedents needed to understand the context and the contents of the Letter.

2) Section 2 in [7] discusses the solutions of Maxwell's Eqs. (1-4) and the Helmholtz equations for the electric intensity and magnetic induction fields Eqs. (5-6), via the integration of their respective sources in the latter with the Green function from Eqs. .(7-8), as expressed in Eqs. (9-10). The second part of the same section, based on [14] in [7], illustrates the decomposition of any field into its longitudinal, toroidal and poloidal components. While the Helmholtz theorem recognizes the decomposition of any vector field into its longitudinal and transverse components, the Debye formalism recognizes that in three dimensions the latter may be of two different types. Equation (11) shows the successive directionality selecting operators: gradient, angular momentum, and rotational of the angular momentum acting on the respective Debye scalar potentials, which are solutions of the homogeneous Helmholtz equation. Equation (12) is the continuity equation connecting the longitudinal current density and the charge density, and its counterpart in Eq. (13) connects the longitudinal Debye potential with the charge density. Equation (14) shows the decomposition of the rotational of the current density into its poloidal and toroidal components, identifiable by their respective operators and the exchange of their Debye scalar potentials. The integrations of Eqs. (9-10) lead to the decompositions of the respective fields Eqs. (15-16) and the identifications of their respective Debye scalar potentials Eqs. (17-22).

We consider now, [8,9] and the Letter, in order to appreciate the changes of going from sources confined on a spherical surface to sources confined on spherical toroidal surfaces. The change of geometry brings in additional boundary conditions, from a single spherical surface to the surfaces of the four sections of the toroidal surface: two spherical and two conical coaxial rings. This explains the spherical symmetry breaking in the Letter, and its consequences in the multipole solutions of the homogeneous Helmholtz equation, as new Debye scalar potentials in Eqs. (1-2) of Sec. 2.

Concerning the vector sources reported in Sec. 3, they are constructed and identified from a common Debye scalar multipole potential, under the action of the successive operators of gradient, which becomes angular momenta when multiplied by the appropriate vectors, and successive rotationals in Eqs. (3, 5-10). The continuity Eq. (4) also yields the charge density. Notice the correspondences and differences with their counterparts in [7].

The construction of the Green functions in Sec. 4, for a unit point source on the toroidal surface, is constructed by the standard method using its expansion in multipole harmonics and spherical Bessel functions of order $\lambda$ of Eqs. (1-2).

The idea of superintegrability and the exact results for spheres [9] and for cylindrical toroids [10] allow us to anticipate the results discussed in Sec. 5. In fact, apart from the different geometries the physical solutions, for the corresponding electric, magnetic and toroidal moments, have common qualitative and quantitative features.

\section{Acknowledgments}

The Authors ELK and HTB gratefully thank the partial financial support provided by Consejo Nacional de Ciencia y Tecnolog Ãa, SNI 1976 and Becas Nacionales 793451, in this work. HTB also acknowledges financial support provided by UNAM-PAPIIT, project IN111820. 
1. V. Savinov, V. A. Fedotov, and N. I. Zheludev, Toroidal dipolar excitation and macroscopic electro- magnetic properties of metamaterials, Physical Review B, vol. 89, (may 2014).

2. N. Papasimakis, V. A. Fedotov, V. Savinov, T. A. Raybould, and N. I. Zheludev, Electromagnetic toroidal excitations in matter and free space, Nature Materials, 15 (2016) 263.

3. N. Talebi, S. Guo, and P. A. van Aken, Theory and applications of toroidal moments in electrodynam- ics: their emergence, characteristics, and technological relevance, Nanophotonics 7 (2018) 93.

4. I. Fernandez-Corbaton, S. Nanz, and C. Rockstuhl, On the dynamic toroidal multipoles from local- ized electric current distributions, Scientifficc Reports 7 (2017).

5. V. A. Fedotov, Electrodynamics beyond common multipoles, in 2019 International Conference on Electromagnetics in Advanced Applications (ICEAA), pp. 0304-0304, (2019).

6. N. Talebi, Near-Field-Mediated Photon-Electron Interactions, ch. Toroidal Moments Probed by Elec- tron Beams. (Springer International Publishing, 2019).
7. A. Góngora T. and E. Ley-Koo, Complete electromagnetic multipole expansion including toroidal moments, Rev. Mex. Fis. E 52 (2006) 177.

8. E. Ley-Koo, A. Góngora T., and H. Torres-Bustamante, Addendum to complete electromagnetic multipole expansion including toroidal moments Rev. Mex. Fis E 52 (2006) 188-197, Rev. Mex. Fis. E 17 (2020) 138.

9. E. Ley-Koo, C. Esparza-López, and H. Torres-Bustamante, Exact solutions for electromagnetic

elds inside and outside a spherical surface with magnetic/electric dipole distributed sources, Rev. Mex. Fis. E 64 (2018) 139.

10. H. Torres-Bustamante and E. Ley-Koo, Puntos cuánticos y radiación electromagnética multipolar en toroides cilindricos, in LXIII Congreso Nacional de Física, Sociedad Mexicana de Fśica, 2020.

11. E. Ley-Koo, Recent progress in confined atoms and molecules: Superintegrability and symmetry breakings, Rev. Mex. Fis. E 64 (2018) 326. 